\title{
Les médecins face à l'événement ultime de la vie
}

«Pleinement en vie, nous sommes entourés par la mort», a écrit un jour Martin Luther. Ce qui, à l'époque du réformateur, résultait de l'expérience quotidienne, aussi en Europe centrale en raison des épidémies, de la mortalité infantile élevée et des guerres, n'est aujourd'hui depuis longtemps plus aussi visible, du moins dans

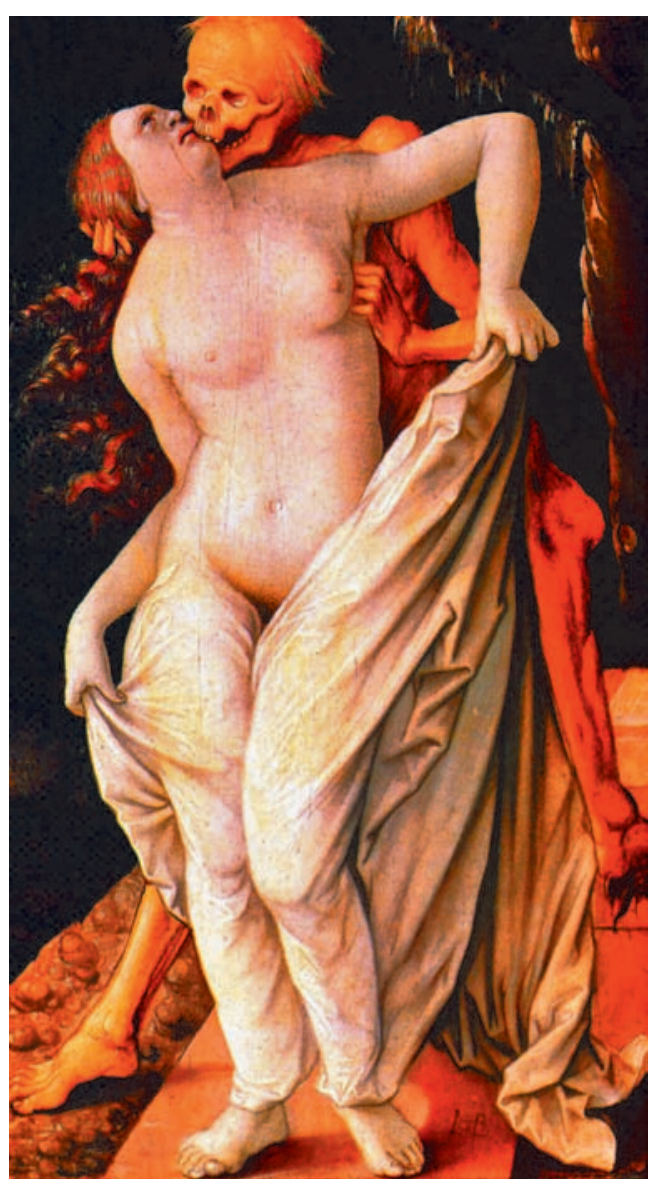

les pays industrialisés. Certes, la mort est omniprésente, de manière virtuelle ou dans les médias (jeux informatiques, films cinématographiques, nouvelles télévisées, etc.). Mais sous nos latitudes, elle reste largement exclue de la vie «réelle», elle est, si possible, toujours bien organisée et se déroule dans des lieux qui lui sont réservés. L'affirmation de Luther reste néanmoins vraie car ce qui vit doit aussi mourir et cet événement peut en principe survenir partout et à n'importe quel moment.

Ce qui vient après la mort relève de la foi, aussi au XXI' siècle: ici, rien n'a changé. Alors que les uns sont convaincus que la vie, dont on ne sait pas exactement ce qu'elle est, encore moins comment elle est apparue, continue sous une forme quelconque, les autres croient que tout est fini et que la mort débouche sur le néant.

Dans ce contexte, on comprend donc aisément l'impact des idées philosophiques et religieuses sur la façon d'envisager $(1518-1520)$ divers et dans une optique différente (l'un concerne la mort du point de vue bouddhiste et les deux autres portent sur l'aide au suicide, qui a été un sujet de controverse au cours de ces derniers mois).

Alors que le temps après la mort ne relève pas de leurs compétences, les médecins sont souvent directement impliqués dans le processus létal. La prise en charge et l'accompagnement des mourants constituent un domaine spécial et l'activité médicale, exigeante, exercée dans ce contexte revêt une importance qui n'est plus à démontrer. De grandes différences demeurent néanmoins dans la question de savoir quel rôle le corps médical doit jouer dans l'aide au suicide.

En règle générale, les médecins sont confrontés à un large éventail d'idées et de convictions quant à l'agonie et à la mort. Compte tenu de ce qui précède, on fait bien d'accueillir l'opinion d'autrui avec respect et sincérité, et pas seulement de façon superficielle, conformément à notre culture médicale et thérapeutique. Cette démarche n'est pas facile car nous avons tous plus ou moins tendance à rabaisser les idées divergentes en considérant les nôtres comme supérieures (par exemple sous le couvert du regret).

Une telle conception n'a rien à voir avec l'indifférence postmoderne: le respect sincère des idées d'autrui présuppose en principe une position personnelle; si tout m'est égal, mon attitude conduit à une pseudo-acceptation dont la base spongieuse va être prise en compte et s'opposer à une relation de confiance véritable, en l'occurrence entre le patient et le médecin. En revanche, si une telle relation est établie, il sera possible de jeter un pont par-dessus les divergences quant à la façon d'envisager la vie et le monde ou du moins à en accepter les conséquences, par exemple dans le cas extrême, le refus d'un traitement médical pour des raisons de conscience.

Bruno Kesseli une mort bonne ou digne. Deux des trois articles publiés dans la présente édition du BMS le confirment de manière impressionnante par l'examen de la mort sous des angles les plus 